\title{
Periosteal Fiber Transection During Periosteal Procedures Is Crucial to Accelerate Growth in the Rabbit Model
}

\author{
Matthew A. Halanski MD, Tugrul Yildirim MD, Rajeev Chaudhary MS, \\ Matthew S. Chin MD, Ellen Leiferman DVM
}

Received: 10 July 2015/Accepted: 19 November 2015/Published online: 15 December 2015

(C) The Association of Bone and Joint Surgeons \& 2015

\begin{abstract}
Background Disruption of the periosteum has been used to explain overgrowth after long bone fractures. Clinically, various periosteal procedures have been reported to accelerate growth with varied results. Differences between procedures and study populations, in these prior studies, make drawing conclusions regarding their effectiveness difficult.

Questions/purposes The purpose of this study was to (1) determine if all reported periosteal procedures accelerate growth and increase the length of bones; (2) study the relative duration of these growth-accelerating effects at two time points; and (3) identify the periosteal procedure that results in the most growth.
\end{abstract}

This study was funded by both the Hartwell Foundation (MAH) and the St Giles Foundation (MAH).

All ICMJE Conflict of Interest Forms for authors and Clinical Orthopaedics and Related Research ${ }^{\mathbb{R}}$ editors and board members are on file with the publication and can be viewed on request.

Clinical Orthopaedics and Related Research ${ }^{\mathbb{R}}$ neither advocates nor endorses the use of any treatment, drug, or device. Readers are encouraged to always seek additional information, including FDAapproval status, of any drug or device prior to clinical use. Each author certifies that his or her institution approved the animal protocol for this investigation and that all investigations were conducted in conformity with ethical principles of research.

This study was conducted at the University of Wisconsin-Madison, Madison, WI, USA.

M. A. Halanski ( $\square)$, T. Yildirim, R. Chaudhary, E. Leiferman Department of Orthopedics \& Rehabilitation, University of Wisconsin-Madison School of Medicine \& Public Health, 1685 Highland Avenue, Madison, WI 53705, USA

e-mail: halanski@ortho.wisc.edu

M. S. Chin

Department of Radiology, Musculoskeletal Division, Johns Hopkins School of Medicine, Baltimore, MD, USA
Methods Periosteal stripping $(\mathrm{N}=8)$, periosteal transection $(\mathrm{N}=8)$, periosteal resection $(\mathrm{N}=8)$, (and) full periosteal release $(\mathrm{N}=8)$ were performed on the tibiae of skeletally immature rabbits. Tibiae were collected 2 weeks postoperatively. The tibiae of additional cohorts of periosteal transection $(\mathrm{N}=8)$, periosteal resection $(\mathrm{N}=8)$, full periosteal release $(\mathrm{N}=8)$, and repetitive periosteal transection $(\mathrm{N}=8)$ were collected 8 weeks postoperatively. The contralateral tibiae served as an operative sham control in all cohorts. Fluorochrome bone labeling was used to measure growth rates, whereas high-resolution Faxitron imaging was performed to measure tibial lengths. Comparisons were then made between (1) experimental and sham controls; and (2) different procedures. Eight additional nonsurgical animals were included as age-matched controls.

Results Growth (in microns) was accelerated at the proximal tibial physis on the tibia undergoing the periosteal surgical procedures versus the contralateral control limb after the transection $(411 \pm 27$ versus $347 \pm 18, \mathrm{p}<0.001$ $[$ mean $\pm \mathrm{SD}])$, resection $(401 \pm 33$ versus $337 \pm 31, \mathrm{p}<$ $0.001)$, and full periosteal release ( $362 \pm 45$ versus $307 \pm$ $33, \mathrm{p}<0.001), 2$ weeks after the index procedure. Conversely, the periosteal stripping cohort trended toward less growth $(344 \pm 35)$ than the controls $(356 \pm 25 ; \mathrm{p}=0.08)$. No differences were found between limbs in the nonoperative controls. Tibial lengths for the experimental tibiae were longer at 2 weeks in the transection $(1.6 \pm 0.4 \mathrm{~mm}, \mathrm{p}$ $<0.001)$, resection $(1.6 \pm 0.9 \mathrm{~mm}, \mathrm{p}=0.03)$, and full periosteal release $(1.7 \pm 0.5 \mathrm{~mm}, \mathrm{p}<0.001)$, whereas negligible differences were found between the tibiae of the nonoperative controls $(0.13 \pm 0.7 \mathrm{~mm}, \mathrm{p}=0.8)$ and stripping cohorts $(0.10 \pm 0.6 \mathrm{~mm}, \mathrm{p}=0.7)$. At 8 weeks, growth acceleration ceased at the proximal tibial physes in the transection cohort $(174 \pm 11$ versus $176 \pm 21, \mathrm{p}=0.8)$, 
and the control limbs actually grew faster than the experimental limbs after resection $(194 \pm 24$ versus $178 \pm 23, p$ $=0.02)$ and full periosteal release $(193 \pm 16$ versus $175 \pm$ $19, \mathrm{p}<0.01)$ cohorts. Growth rates were increased over control limbs, only in the repetitive transection cohort (190 \pm 30 versus $169 \pm 19, \mathrm{p}=0.01$ ) at 8 weeks. Tibial lengths for the experimental tibiae remained longer at 8 weeks in the transection $(1.4 \pm 0.70 \mathrm{~mm}, \mathrm{p}<0.001)$, resection $(2.2$ $\pm 0.82 \mathrm{~mm}, \mathrm{p}<0.001)$, full periosteal release $(1.6 \pm 0.42$ $\mathrm{mm}, \mathrm{p}<0.001)$, and repetitive periosteal transection $(3.3 \pm$ $1.1 \mathrm{~mm}, \mathrm{p}<0.001$ ), whereas negligible differences were found between the tibiae of the nonoperative controls $(-0.08 \pm 0.58 \mathrm{~mm}, \mathrm{p}=0.8)$. Comparing the procedures at 2 weeks postoperatively, no differences were found in tibial lengths among the transection $(2.1 \% \pm 0.5 \%$ increase), resection $(2.1 \% \pm 1.1 \%$ increase), and full periosteal release $(2.1 \% \pm 0.6 \%)$; however, all three demonstrated greater increased growth when compared with the stripping cohort $(-0.10 \% \pm 0.7 \%$; $<<0.05)$. At 8 weeks no differences could be found between increased tibial lengths among the transection (1.5\% $\pm 0.7 \%)$, resection $(2.3 \% \pm 0.9 \%)$, and full periosteal release $(1.7 \%$ $\pm 0.4 \%$ ). The repetitive transection produced the greatest over length increase $(3.5 \% \pm 1 \%)$, and this was greater than the acceleration generated by the single resection $(\mathrm{p}<$ $0.001)$ or the full periosteal release $(p=0.001)$. All four demonstrated an increase greater than the nonoperative control $(0.09 \% \pm 0.6 \% ; \mathrm{p}<0.05)$.

Conclusions Transection of the longitudinally oriented periosteal fibers appears critical to accelerate growth in a rabbit model.

Clinical Relevance These findings in an animal model support previous claims that limb overgrowth occurs as the result of periosteal disruption. Based on these findings in rabbits, we believe that less invasive procedures like periosteal transection are a promising avenue to explore in humans; clinical studies should seek to determine whether it is equally effective as more invasive procedures and its role as an adjunct to guided growth or distraction osteogenesis.

\section{Introduction}

Many pediatric orthopaedic procedures are performed to correct the effects of abnormal skeletal growth. Spine and limb deformities, hip dysplasia, and limb length discrepancies are childhood disorders rooted in abnormal skeletal growth. Currently, these conditions are treated using invasive procedures that decrease (guided growth), eliminate (fusions and epiphysiodesis), or ignore (osteotomies) potential growth that could be harnessed to correct the deformity. Periosteal procedures (stripping, transection, and resection) are the only known surgical procedures that have demonstrated the ability to accelerate growth. Differences between these procedures and the mechanism by which they accelerate growth are poorly understood.

This lack of knowledge has led to different procedures being performed in each study using various models with often conflicting results. Growth acceleration has been found to occur in many animal species after periosteal resection (cutting and removal of a circumferential strip of periosteum) [8, 16, 20, 28, 32]. However, the procedure of periosteal stripping (detaching the periosteum from the underlying bone without circumferentially cutting or removing the periosteum) has also been shown to accelerate physeal growth $[1,9,10,13,25]$. The two papers that demonstrate the most consistent and promising clinical results combined both circumferential periosteal transection with periosteal stripping or resection [7, 15]. Thus, knowing which of these procedures will yield the most growth with the least morbidity is unknown and has likely contributed to their lack of clinical use.

The purpose of this study was to critically evaluate growth rates after four periosteal procedures using a single animal model. Specifically we set out to (1) determine if all reported periosteal procedures accelerate growth and increase the length of bones; (2) study the relative duration of these growth accelerating effects at two time points; and (3) identify the periosteal procedure that results in the most growth. Our hypothesis was that (1) proximal tibial growth rates; and (2) overall tibial lengths would increase with increasing periosteal insult (invasiveness). Growth rates, using fluorochrome labeling, and overall bone lengths, using high-resolution radiography, were used in a rabbit model to test these hypotheses at 2 and 8 weeks postprocedure.

\section{Materials and Methods}

The following study was approved by the appropriate local institutional animal care and use committee.

Animals and Power Analysis

We chose a rabbit model for this study because the authors found the size of the rabbit, in a prestudy evaluation, to be the smallest readily available laboratory mammal available that allowed the different surgical procedures to be performed in a reproducible manner. Although similar previous work has been done at our institution using lambs [22], the cost associated with using large number of animals required for this study was prohibitive. Therefore, 
7- to 8-week-old female, New Zealand White rabbits obtained from commercial suppliers, Envigo (formerly Harlan) from their breeding facility in Oxford, MI, USA, and from Charles River Laboratories, from their breeding facility in Canada, weighing between 1 and $2 \mathrm{~kg}$ were used in this study. Based on previously obtained growth data, a power analysis was performed estimating that the procedures would demonstrate a $10 \%$ difference in growth rate between experimental and sham control limbs. Using these numbers, at least eight subjects were needed in each procedural cohort for each time period ( 2 and 8 weeks) to give $80 \%$ power. Thus, a total of 64 operative animals, each with an experimental and sham (internal control) limb, were used, eight animals for each procedure performed at each time point. An additional eight nonoperative animals were used as external nonsurgical controls age-matched to include three with the 2-week followup and five with the 8 -week followup. These animals were administered pulsed bone labels within 5 days of euthanasia to provide normal rates of growth in nonoperative animals.

All rabbits were acclimated to the animal care facility for at least 5 to 7 days, were "approved for use on study" by the facility veterinarian, and then underwent the designated surgical procedure. Baseline comparisons were made of the size and age of animals used in each cohort with respect to the 2-week (Table 1) and 8-week (Table 2) periods. Experiments demonstrated that although these cohorts were relatively uniform, small differences were found. Minimal differences in age at euthanasia were noted (and therefore at the time of surgery because all animals were euthanized 14 or 56 days postoperatively). The largest difference in age between cohorts was less than 6 days. Similarly, several operative cohorts gained more weight than the nonoperative controls.

\section{Surgical Procedures}

The investigators adapted and standardized four procedures for use in our rabbit model based off of the various procedures previously described in animal models and human studies. For each procedure, a longitudinal medial skin incision was made over both the right and left proximal tibial regions, approximately 1 to $2 \mathrm{~cm}$ in length. Muscles were elevated off the control tibia causing minimal disturbance to the underlying periosteum (sham/control), whereas the experimental side underwent various periosteal release techniques. In all techniques, proximal periosteal disruption was limited to below the visible distal medial collateral ligament (MCL) insertion. The four (five

Table 1. Comparison of cohorts in 2-week experiments

\begin{tabular}{llllll}
\hline Procedure & 2 weeks (number) & Initial weight $(\mathrm{kg})$ & Weight at 2-week euthanasia & Weight gain $(\mathrm{kg})$ & Age (days) at euthanasia* \\
\hline Negative control & 3 & $1.03 \pm 0.04$ & $1.35 \pm 0.08$ & $0.32 \pm 0.12$ & $62 \pm 0^{\dagger}$ \\
Periosteal stripping & 8 & $0.92 \pm 0.06$ & $1.40 \pm 0.10$ & $0.49 \pm 0.10$ & $64 \pm 0^{\dagger, *}$ \\
Periosteal transection & 8 & $1.04 \pm 0.09$ & $1.55 \pm 0.11$ & $0.50 \pm 0.13$ & $61.8 \pm 1.7^{\dagger, *}$ \\
Periosteal resection & 8 & $1.02 \pm 0.13$ & $1.48 \pm 0.17$ & $0.46 \pm 0.12$ & $61.6 \pm 1.5^{\dagger, *}$ \\
Full periosteal release & 8 & $1.23 \pm 0.09$ & $1.61 \pm 0.12$ & $0.38 \pm 0.12$ & $67.0 \pm 0.0^{\dagger}$ \\
Periosteal transection $\times 2$ & NA & NA & NA & NA & NA \\
\hline
\end{tabular}

Analysis of variance performed between cohorts followed by pairwise Tukey's analysis to assess differences in weight and age; all values are averages $\pm \mathrm{SD} ; *$ analysis of variance $<0.001 ;{ }^{\dagger} \mathrm{p}<0.05$ between greatest value and other cohorts; ${ }^{\star} \mathrm{p}<0.05$ between greatest value and other cohorts; NA $=$ not applicable because this cohort was not tested at this time point.

Table 2. Comparison of cohorts in 8-week experiments

\begin{tabular}{llllll}
\hline Procedure & 8 weeks (number) & Initial weight $(\mathrm{kg})$ & Weight at 8 -week euthanasia & Weight gain $(\mathrm{kg}) *$ & Age (days) at euthanasia \\
\hline Negative control & 5 & $1.02 \pm 0.16$ & $2.30 \pm 0.19$ & $1.28 \pm 0.17^{\dagger}$ & $103 \pm 1.41^{\dagger, *}$ \\
Periosteal stripping & NA & NA & NA & NA & NA \\
Periosteal transection & 8 & $1.02 \pm 0.08$ & $2.47 \pm 0.15$ & $1.45 \pm 0.09^{\dagger}$ & $104.5 \pm 1.69^{\dagger}$ \\
Periosteal resection & 8 & $1.05 \pm 0.08$ & $2.54 \pm 0.21$ & $1.49 \pm 0.17$ & $104.1 \pm 1.81^{\dagger}$ \\
Full periosteal release & 8 & $1.02 \pm 0.13$ & $2.64 \pm 0.25$ & $1.62 \pm 0.15 \dagger$ & $105.8 \pm 0.71^{\dagger}$ \\
Periosteal transection $\times 2$ & 8 & $1.06 \pm 0.12$ & $2.43 \pm 0.10$ & $1.38 \pm 0.13$ & $106.8 \pm 0.46^{\dagger}$ \\
\hline
\end{tabular}

Analysis of variance performed between cohorts followed by pairwise Tukey's analysis to assess differences in weight and age; all values are averages $\pm \mathrm{SD} ; *$ analysis of variance $<0.01 ;{ }^{\dagger} \mathrm{p}<0.05$ between greatest value and other cohorts; ${ }^{*} \mathrm{p}<0.05$ between greatest value and other cohorts; NA = not applicable because this cohort was not tested at this time point. 

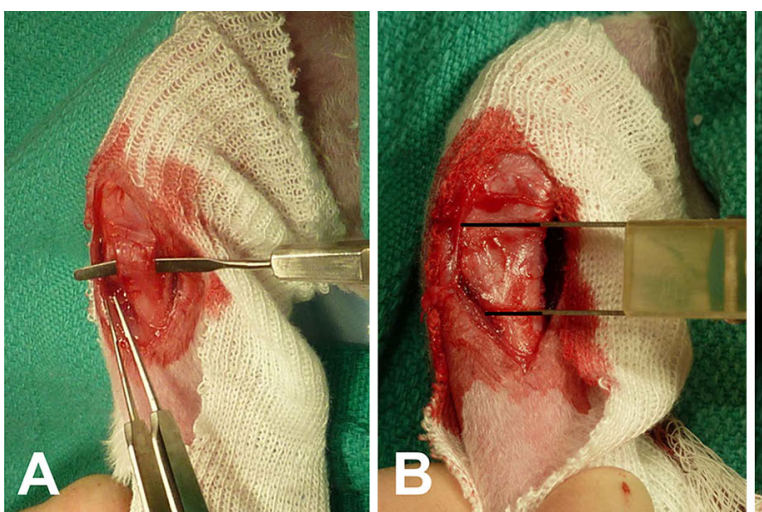

Fig. 1A-D Periosteal procedures were performed in this study. (A) Periosteal stripping: the periosteum is elevated but longitudinal fibers are not cut. (B) Periosteal transection: periosteal fibers are cut circumferentially just distal to MCL insertion and at a point $10 \mathrm{~mm}$ distal. (The identical procedure was performed twice, at time $=0$ and time $=4$ weeks in the repetitive periosteal transection cohort.) $(\mathbf{C})$

including the repetitive transection) procedures performed are described subsequently (Fig. 1).

\section{Periosteal Stripping}

Three approximately 1-cm longitudinal incisions in the periosteum were performed at the three apices of the tibia. A periosteal elevator was then inserted to carefully elevate the periosteum between these incisions without tearing it. Thus, the continuity of longitudinal fibers of the periosteum remained intact.

\section{Periosteal Transection}

Two parallel, circumferential incisions were made in the periosteum around the proximal tibia. These incisions were perpendicular to the long axis of the tibia, separated by 1 $\mathrm{cm}$, using a custom-designed dual-bladed scalpel. The parallel incisions transected the longitudinal periosteal fibers just below the MCL insertion and at a point $1 \mathrm{~cm}$ distal. The intervening periosteum, between the parallel incisions, was left undisturbed.

\section{Periosteal Resection}

Using the same custom two-bladed scalpel, identical periosteal incisions as described previously for the periosteal transection were performed; however, in this cohort, the intervening periosteum was removed as a circumferential periosteal strip $10 \mathrm{~mm}$ wide.

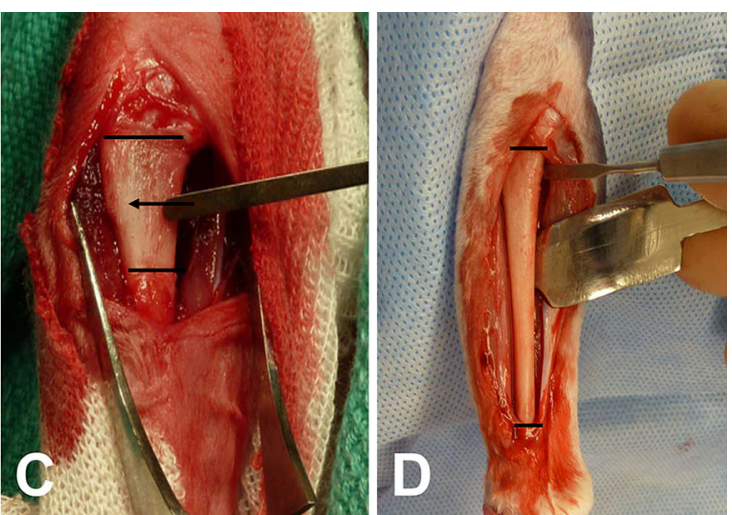

Periosteal resection is the same as (B) but the intervening strip of periosteum is removed (arrow pointing to denuded bone). (D) Full periosteal release: the periosteum is circumferentially elevated the length of the bone and then circumferentially transected just distal to the MCL insertion and proximal to the distal tibial physis.

\section{Full Periosteal Release}

The surgical approach in this cohort required a longer anterior incision, approximately the full length of the tibia. The periosteal fibers were transected circumferentially both proximally (just below the level of the MCL insertion) and as far distally as possible, although proximal to the distal tibial growth plate. An additional longitudinal incision in the periosteum was made connecting the two transverse transections. The entire tibial periosteum was then elevated but not removed.

\section{Repetitive Periosteal Transection}

An identical surgical procedure was used as described for the periosteal transection at time zero. Four weeks after this initial procedure, an identical procedure was performed on the same limb. Briefly, two parallel, circumferential incisions were made in the periosteum around the proximal tibia. These incisions were perpendicular to the long axis of the tibia, separated by $1 \mathrm{~cm}$, using a custom-designed dualbladed scalpel. The parallel incisions transected the longitudinal periosteal fibers just below the MCL insertion and at a point $1 \mathrm{~cm}$ distal. The intervening periosteum, between the parallel incisions, was left undisturbed.

\section{Outcome Measures}

Tibial growth rates and overall tibial lengths were the primary outcomes measured as described subsequently. The primary comparisons made in this study evaluated the differences in tibial growth rates and lengths between the experimental 
(periosteal procedure) limbs and the operative sham (internal control) limbs of each animal, for each cohort, at each time point. Secondary comparisons between of each of the procedures were made comparing the growth rates and changes in lengths of the experimental limbs from each cohort. Nonoperative, external controls were included for further comparison, because the anesthesia and surgical procedures may have had some unrecognized effect on growth.

\section{Growth Rates}

Fluorochrome Bone Labeling, Tissue Processing, and Histology The antibiotic-derived oxytetracycline $(2 \mathrm{mg} / \mathrm{kg}$ IV) and the plant dye, alizarin complexone (15 mg/kg IV), were used for pulsed fluorochrome labeling in this study. Alizarin was given 96 hours and oxytetracycline 24 hours preeuthanasia. After euthanasia, the femurs and tibiae of the rabbits were collected and fixed in $70 \%$ ethanol solution. Bones were then coronally sectioned into 1-mm thick slabs using an Isomet Precision saw (Buehler Isomet 2000, Lake Bluff, IL, USA) and visualized using a Nikon Optiphot (Nikon Instruments, Melville, NY, USA) microscope setup for epifluorescence. Alizarin complexone was optimally viewed with a 510- to 560-nm excitation filter and 590-nm barrier filter, whereas oxytetracycline was viewed optimally with a 405-nm excitation filter and 470-nm barrier filter (Fig. 2A). Measurements were made at 2-mm intervals across the width of each growth plate excluding the $2 \mathrm{~mm}$ closest to perichondrium to reduce effects of the groove of Ranvier (Fig. 2B). These measurements were averaged to give a final growth rate. Growth rates of the distal femur, proximal tibia, and distal tibia for the experimental and control limbs were measured for each animal.

\section{Tibial Lengths}

Final Tibial Length Assessment After harvest, the soft tissues surrounding the tibiae were removed. The bones were then optimally positioned and imaged using high-resolution Faxitron (Hewlett Packard, Tucson, AZ, USA) imaging (Fig. 3). The film was then digitized and measurements made using Photoshop C3 Extended (Adobe, San Jose, CA, USA). Length was determined by measuring between the tibial eminences proximally to the distal tibial plafond distally.

\section{Statistical Analysis}

The growth rates and tibial lengths of each animal were recorded. Differences in growth rates and tibial lengths
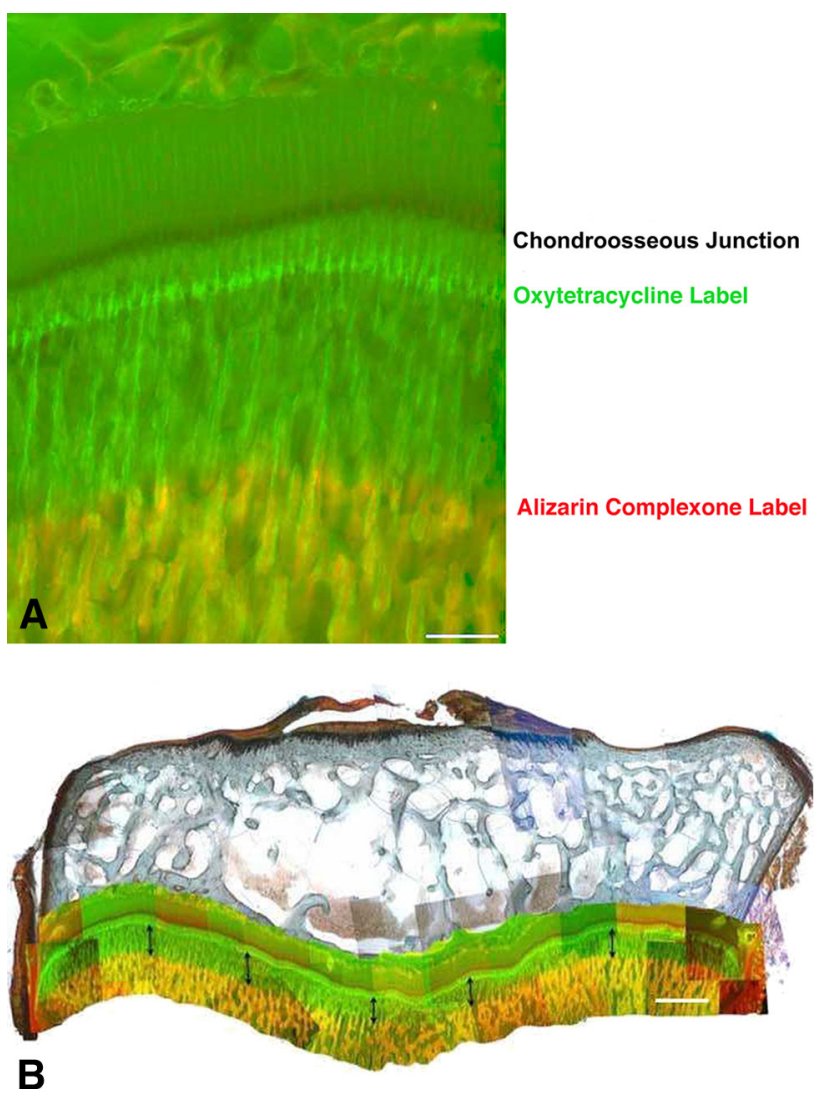

Fig. 2A-B Examples of fluorochrome bone labeling are shown. (A) By pulsing the bone at set times with two different fluorochrome labels (oxytetracycline and alizarin complexone) and measuring the distance between the labels, the rate of bone growth can be calculated. Scale bar $=200 \mu \mathrm{m}$. (B) Montage of proximal tibia: arrows demonstrate the distance between the two bone labels across the tibia. Scale bar $=1 \mathrm{~mm}$

between experimental and sham limbs were compared. Absolute and percentage differences in growth rates were calculated for each animal at each growth plate and these were used to compare the relative effects of growth acceleration between procedures. Paired Student's t-tests were used to compare the daily growth rate through the fluorochrome labeling between the operative and sham limbs. An analysis of variance was performed to determine any significant difference in growth rates among the procedures. When a significant difference was found, a Tukey's pairwise post hoc analysis was performed. A p value $<0.05$ was considered statistically significant. Statistics were performed in KaleidaGraph (Synergy Software, Reading, PA, USA).

\section{Results}

Growth (in microns) was accelerated at the proximal tibial physis on the tibia undergoing the periosteal surgical 


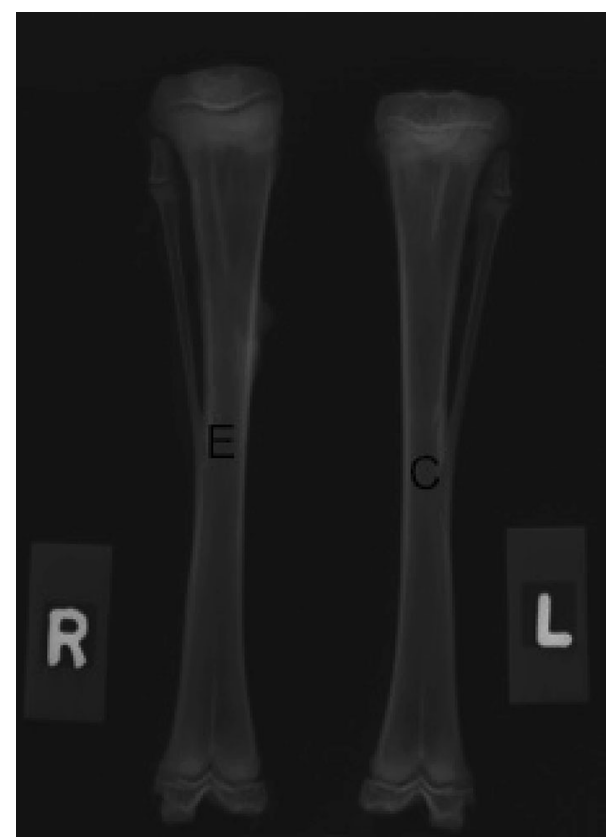

Fig. 3 Example of Faxitron image used to measure tibial lengths. In this example the right tibia $(\mathrm{R})$ had undergone periosteal resection, whereas the left $(\mathrm{L})$ was the sham control.

procedures versus the contralateral control limb for the transection $(411 \pm 27$ versus $347 \pm 18, \mathrm{p}<0.001$ [mean \pm $\mathrm{SD}])$, resection ( $401 \pm 33$ versus $337 \pm 31, \mathrm{p}<0.001)$, and full periosteal release $(362 \pm 45$ versus $307 \pm 33$, p < 0.001 ) cohorts 2 weeks after the index procedure. Conversely, the periosteal stripping cohort trended toward less growth $(344 \pm 35)$ than the contralateral controls $(356 \pm$ $25 ; \mathrm{p}=0.08)$, whereas no differences were found between limbs in the nonoperative controls. Surprisingly, growth was also accelerated on the experimental limbs at the distal tibial physis 2 weeks postoperatively after stripping ( $266 \pm$ 26 versus $248 \pm 21, \mathrm{p}=0.02)$, transection ( $301 \pm 25$ versus $249 \pm 17, \mathrm{p}=0.001)$, resection $(303 \pm 39$ versus $248 \pm 25$, $\mathrm{p}<0.001)$, and full periosteal release $(231 \pm 39$ versus 187 $\pm 37, \mathrm{p}<0.001$ ) (Table 3). No differences in the distal femoral growth rates were found between limbs in any cohort (data not shown). Tibial lengths for the experimental tibiae were longer at 2 weeks in the transection $(1.6 \pm 0.4$ $\mathrm{mm}, \mathrm{p}<0.001)$, resection $(1.6 \pm 0.9 \mathrm{~mm}, \mathrm{p}=0.03)$, and full periosteal release $(1.7 \pm 0.5 \mathrm{~mm} ; \mathrm{p}<0.001)$, whereas negligible differences were found between the tibiae of the nonoperative controls $(0.13 \pm 0.7 \mathrm{~mm}, \mathrm{p}=0.8)$ and stripping cohorts $(0.10 \pm 0.6 \mathrm{~mm}, \mathrm{p}=0.7)$. Because the periosteal stripping cohort exhibited only minimal growth acceleration at the distal tibial physis and no significant increase in length at 2 weeks, the authors abandoned carrying out a second cohort of periosteal stripping animals to 8 weeks. Instead, the cohort undergoing repetitive transection was substituted in its place as one of the cohorts in the 8-week followup group.

At 8 weeks, growth acceleration ceased at the proximal tibial physis in the transection cohort $(174 \pm 11$ versus 176 $\pm 21, \mathrm{p}=0.8$ ), and the control limbs actually grew faster than the experimental limbs after resection $(194 \pm 24$ versus $178 \pm 23, p=0.02)$ and full periosteal release (193 \pm 16 versus $175 \pm 19, \mathrm{p}<0.01)$ cohorts. Growth acceleration was increased over controls at the proximal tibia at 8 weeks only in the repetitive transection cohort $(190 \pm 30$ versus $169 \pm 19, p=0.01$ ). Surprisingly, growth remained accelerated at the distal tibia physis for all of the experimental limbs: transection $(98 \pm 16$ versus $84 \pm 16, \mathrm{p}<$ $0.001)$, resection $(103 \pm 19$ versus $90 \pm 18, p=0.04)$, full periosteal release $(98 \pm 12$ versus $81 \pm 9.0, \mathrm{p}<0.001)$, and repetitive periosteal transection $(96 \pm 17$ versus $73 \pm$ $16, \mathrm{p}<0.001)$. No differences in the distal femoral growth rates were found between limbs in any cohort (data not shown). Tibial lengths for the experimental tibiae were longer at 8 weeks in the transection $(1.4 \pm 0.70 \mathrm{~mm}, \mathrm{p}<$ $0.001)$, resection $(2.2 \pm 0.82 \mathrm{~mm}, \mathrm{p}<0.001)$, full periosteal release $(1.6 \pm 0.42 \mathrm{~mm}, \mathrm{p}<0.001)$, and repetitive periosteal transection $(3.3 \pm 1.1 \mathrm{~mm}, \mathrm{p}<0.001)$, whereas negligible differences were found between the tibiae of the nonoperative controls $(-0.08 \pm 0.58, \mathrm{p}=0.8)$ (Table 4).

Comparisons between procedures (given as percent increase over controls) demonstrated no differences among transection $(18.6 \% \pm 8.7 \%$ increase), resection $(19.0 \% \pm$ $5.9 \%$ increase), and full periosteal release $(18.0 \% \pm 8.0 \%$ increase) at the proximal tibial growth 2 weeks postoperatively. All three of these procedures demonstrated greater growth acceleration compared with the stripping $(-3.50 \%$ $\pm 4.9 \%$ decrease) and nonoperative cohorts at the proximal physis $(-1.03 \% \pm 2.1 \%$; $\mathrm{p}<0.05)$ for each pairwise comparison. At the distal tibia, no differences in growth acceleration were observed among transection $(20.7 \% \pm$ $7.3 \%$ increase), resection ( $21.8 \% \pm 4.8 \%$ increase), and full periosteal release $(24.4 \% \pm 12 \%$ increase); however, all three cohorts demonstrated more growth when compared with the stripping $(7.20 \% \pm 6.9 \%$ increase $)$ and nonoperative cohorts $(-1.78 \% \pm 6.6 \%$ decrease; $p<0.5)$ for each pairwise comparison. At 2 weeks postoperatively, no differences could be found in tibial lengths among the transection $(2.1 \% \pm 0.5 \%$ increase), resection (for a $2.1 \%$ $\pm 1.1 \%$ increase), and full periosteal release (for a $2.1 \% \pm$ $0.6 \%)$; however, all three demonstrated greater increased growth when compared with the stripping cohort $(-0.10 \%$ $\pm 0.7 \% ; \mathrm{p}<0.05)$. Comparisons among procedures at 8 weeks demonstrated no differences among transection $(-0.2 \% \pm 9.3 \%)$, resection $(-7.7 \% \pm 6.1 \%)$, and full periosteal release $(-9.4 \% \pm 5.2 \%)$ because the experimental limbs actually grew slower at the proximal tibia than the control limbs; however, the repetitive transection 

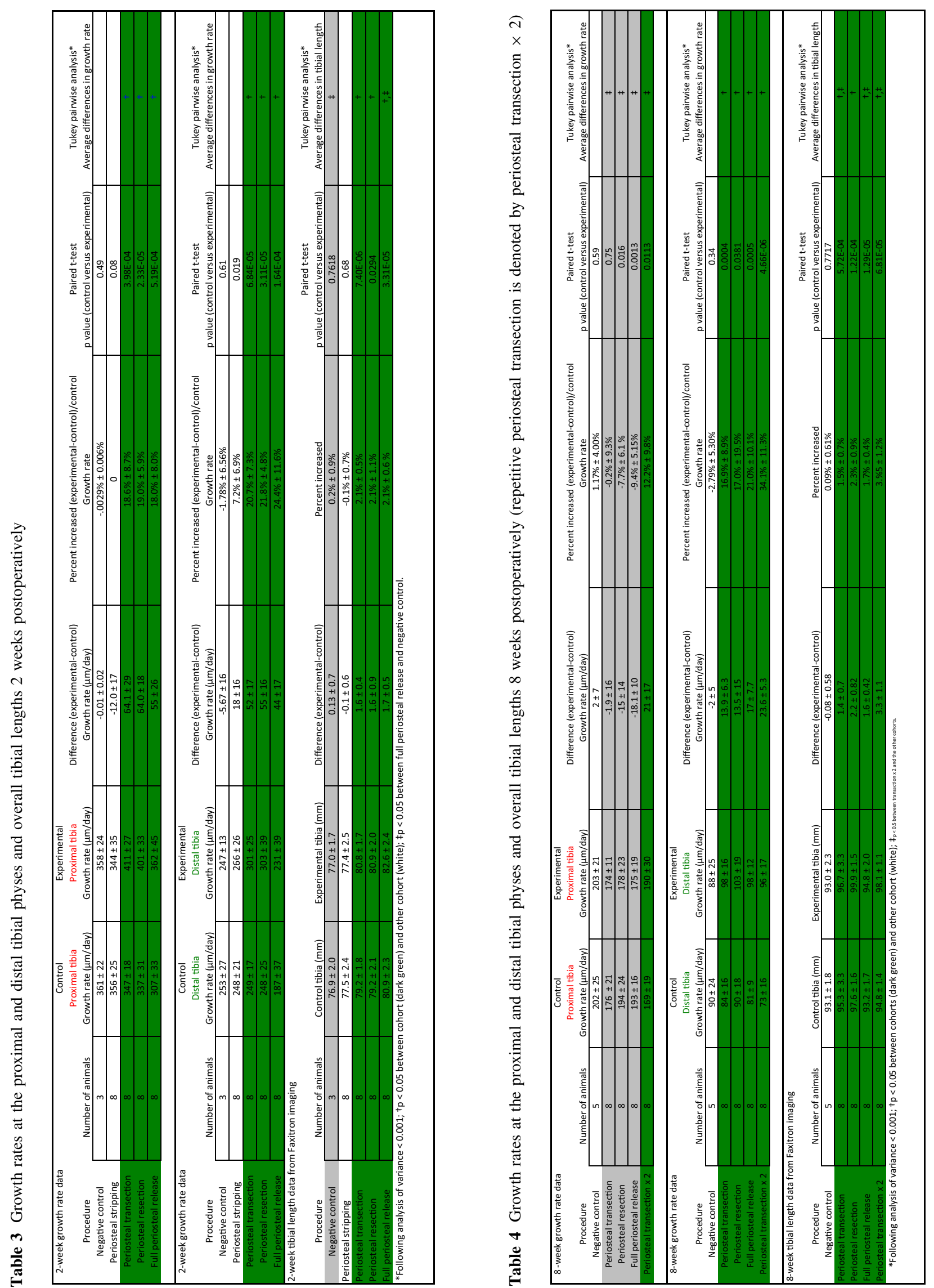
resulted in greater growth $(12.2 \% \pm 9.8 \%)$ than these three other procedures $(p<0.05)$. No difference was found among the procedures at the distal tibia; however, all periosteal procedures demonstrated greater growth acceleration, transection $(16.9 \% \pm 8.9 \%)$, resection $(17.0 \% \pm$ $19 \%)$, full release $(21.0 \% \pm 10 \%)$, and repeated transection $(34.1 \% \pm 11 \%)$ compared with the nonoperative cohorts at the proximal physis $(-2.79 \% \pm 5.3 \% ; \mathrm{p}<0.05)$ for all pairwise comparisons with the nonoperative cohort. No differences could be found between increased tibial lengths among the transection $(1.5 \% \pm 0.7 \%)$, resection $(2.3 \% \pm$ $0.9 \%)$, and full periosteal release $(1.7 \% \pm 0.4 \%)$. The repetitive transection produced the greatest over length increase $(3.5 \% \pm 1 \%)$, which was greater than the acceleration generated by the single resection $(\mathrm{p}<0.001)$ or the full periosteal release $(p=0.001)$, but only trended toward being greater when compared with resection $(p=0.06)$. All four demonstrated an increase greater than the nonoperative control $(0.09 \% \pm 0.6 \% ; \mathrm{p}<0.05)$.

\section{Discussion}

Growth acceleration has previously been demonstrated after periosteal procedures. Although some have reported accelerated growth after stripping [1, 9, 10, 13, 25], more often growth acceleration has been reported after periosteal transection [16, 19, 31] and resection [8, 16, 20, 28, 32]. Our observed growth acceleration after periosteal transection, resection, full periosteal release, and repetitive transection appears similar to that reported in such studies (10\%-30\% over control limbs) [16]. By comparing the different procedures head to head in the same mammalian model, our results most clearly demonstrate that it is the transverse transection of the longitudinal periosteal fibers that appears to be the most important aspect in accelerating growth.

The largest limitation of our study is the use of the rabbit model, because this raises the question of whether these findings apply to clinical practice. The rabbit was chosen over rodent models, because preliminary work determined that the rabbit's size was as small as we felt technically feasible to ensure a high degree of reproducibility in performing the periosteal procedures. Larger and perhaps more clinically applicable models such as the pig or lamb were not chosen because these were not economically feasible options given the high number of animals necessary to adequately power this study. Thus, given the limitations of our model, final conclusions should be limited to the rabbit until further confirmation of these findings in larger animals is performed. Another lesser limitation was small preoperative differences between cohorts (Tables 1, 2). Despite best attempts to minimize differences between animals in each cohort, small differences ( $<6$ days of age and $<200 \mathrm{~g}$ weight gain) were found. These minor differences likely do not affect the overall message from this work.

Additional limitations include the lack of differences in overall final tibial lengths among most of the periosteal procedures, which may be a function of this study being powered to determine a difference in proximal tibial growth rates (experimental versus sham) rather than length among procedures. The difference that was found between the repetitive transection cohort and the others, excluding the resection group $(p=0.06)$, shows the additive effects of repeated procedures on growth. Another potential limitation may include not carrying the periosteal stripping cohort out to 8 weeks postoperatively. However, this cohort exhibited only minimal growth acceleration at the distal tibial physis and no increase in proximal growth acceleration and tibial length at 2 weeks. From previous findings in lambs [22], decreased growth rates over time found in this study, and other data not included in this study, growth accelerating after these periosteal procedures is transient, decreasing over time. With no appreciable increase in overall growth at 2 weeks, the authors felt the cost and lives of the additional animals would be better used in evaluating the effects of repeating transection. Finally, our nonoperative controls appeared to be growing slightly faster than the nonoperative shams. Although these differences were not large, they do work to exaggerate the differences between experimental and control limbs. Although nonoperative internal controls could have been used, the authors felt that the sham control offered the best internal control to minimize confounding factors such as pain, inflammation, and differential weightbearing. It is difficult to know whether the apparent decrease in growth on the operative sham is the result of the stress of anesthesia or a process with the body slowing down growth on one side while speeding it up on the other. A nonoperative internal limb may be included in future studies to determine this answer.

Although previous studies have reported similar findings $[8,16,20,28,32]$, we believe the current study is the most comprehensive study to date to critically compare all the clinically relevant periosteal techniques in a single, mammalian model. Our use of pulsed fluorochrome labeling allowed us to track growth rates after these procedures over time. This technique yielded perhaps one of our most interesting findings. The proximal tibial periosteal procedures resulted in growth acceleration at the distal tibial growth plate, and the accelerated growth lasted longer distally than it did proximally. These findings are similar to those reported by Crilly [5], who reported this finding in chick radii, but to our knowledge, this phenomenon has not been reported in mammals until now. Previously, repetitive 
full periosteal stripping (similar to our full periosteal release cohort) had been shown to increase longitudinal growth in the dog and chimpanzee models when compared with a single procedure [25], similar to our findings of additional growth after repetitive periosteal transection. The morbidity associated with the full periosteal stripping procedure and the relatively high risk of postoperative fractures $(25 \%-50 \%)$ precluded clinical translation of these previous findings. However, our findings that repeating simple transection resulted in similar or more growth may provide an opportunity to develop novel, clinically relevant, less invasive techniques to harness this growth potential.

Previous authors have attributed growth acceleration after periosteal procedures to a mechanical detethering of the physis $[5,8,11,13,14,24,29,31,33,34]$. This mechanism is plausible in that more growth acceleration occurred after transection, resection, and full release than with the stripping procedure (where the longitudinal periosteal fibers remained intact). This is further supported by findings that the increased growth rates were very similar regardless of the procedure performed. Thus, it appears that the physis has a set rate at which it can grow after the restraint of the periosteum is released. The mechanical properties of the periosteum would also support this hypothesis, because the periosteum's fibers appear strong enough to retard growth. Mechanical studies of the periosteum in several species demonstrate ultimate tensile strength of over $25 \mathrm{MPa}[2,18,30]$ and resting tensions in the order of $0.92 \mathrm{MPa}$ [2]. These strengths are well above the $0.1 \mathrm{MPa}$ found to decrease physeal growth on the order of $10 \%$ to $20 \%$ [27]. Thus, it is reasonable to infer that releasing this tensile structure could reduce the compressive forces across the growth plate resulting in accelerated growth.

Alternative explanations for our findings include that the growth acceleration is the result of increased local inflammation or blood flow. Although we did not completely rule out this possibility, the fact that periosteal stripping resulted in minimal growth, accelerated growth occurred distally (remotely) for an extended period of time, and more soft tissue injury did not result in significantly greater growth rates all decrease the likelihood of this explanation. We presume that the small differences in ultimate tibial length among three procedures may be the result of differences in the duration of time that it takes to heal the periosteal injury. Once the periosteum is repaired, the acceleration ceases. The additional growth that is observed after repetitive transections supports this presumption, and future work is aimed at confirming this hypothesis.

Although clinical translation of any findings in an animal model must be done cautiously, this study may help explain the periosteum's role in the common clinical phenomenon of limb overgrowth after long bone fracture $[3,6]$ and Cozen's effect (genu valgum after nondisplaced proximal tibial fracture) $[4,12,26]$ in pediatric patients. Our results support the idea that it is the disruption of the longitudinal periosteal fibers at the time of the injury that determines whether overgrowth occurs. Also, our findings suggest that periosteal transection might be useful in offsetting the growth inhibition that is reported after traditional tibial limb lengthening procedures [21, 23]. Similarly, the ability to accelerate growth through these procedures may prove useful as a simple adjunct to other current therapies reliant on growth such as "guided growth" procedures [17]. However, all of those contentions would need to be tested in larger mammal models that more closely emulate the mechanisms of human bone growth and then, if they still look promising, in human studies.

In conclusion, growth acceleration occurs after multiple periosteal transection procedures in the rabbit tibia model but does not occur after simple periosteal stripping. Growth acceleration was found to occur at both the proximal and distal growth plates after proximal periosteal procedures. Rates of accelerated growth were similar after periosteal transection, resection, and full periosteal release, but appear short-lived. Based on these findings in rabbits, we believe that less invasive procedures like periosteal transection is a promising avenue to explore in humans. Clinical studies should seek to determine its effectiveness as more invasive procedures and a potential role as an adjunct to guided growth or distraction osteogenesis. Future studies should evaluate this proposition in larger animal models and, if those prove promising, in human clinical studies.

Acknowledgments We thank Dr Kenneth J. Noonan for inspiring us to pursue this line of inquiry.

\section{References}

1. Bertone AL, Turner AS, Park RD. Periosteal transection and stripping for treatment of angular limb deformities in foals: clinical observations. J Am Vet Med Assoc. 1985;187:145-152.

2. Bertram JE, Polevoy Y, Cullinane DM. Mechanics of avian fibrous periosteum: tensile and adhesion properties during growth. Bone. 1998;22:669-675.

3. Buckley SL, Smith G, Sponseller PD, Thompson JD, Griffin PP. Open fractures of the tibia in children. J Bone Joint Surg Am. 1990;72:1462-1469.

4. Cozen L. Fracture of the proximal portion of the tibia in children followed by valgus deformity. Surg Gynecol Obstet. 1953;97:183-188.

5. Crilly RG. Longitudinal overgrowth of chicken radius. J Anat. 1972;112:11-18.

6. Czertak DJ, Hennrikus WL. The treatment of pediatric femur fractures with early 90-90 spica casting. J Pediatr Orthop. 1999;19:229-232. 
7. D'Souza H, Shah NM. Circumferential periosteal sleeve resection: results in limb-length discrepancy secondary to poliomyelitis. $J$ Pediatr Orthop. 1999;19:215-221.

8. Dimitriou CG, Kapetanos GA, Symeonides PP. The effect of partial periosteal division on growth of the long bones. An experimental study in rabbits. Clin Orthop Relat Res. 1988;236:265-269.

9. Garces GL, Hernandez Hermoso JA. Bone growth after periosteal stripping in rats. Int Orthop. 1991;15:49-57.

10. Hernandez JA, Serrano S, Marinoso ML, Aubia J, Lloreta J, Marrugat J, Diez A. Bone growth and modeling changes induced by periosteal stripping in the rat. Clin Orthop Relat Res. 1995;320:211-219.

11. Houghton GR, Dekel S. The periosteal control of long bone growth. An experimental study in the rat. Acta Orthop Scand. 1979;50:635-637.

12. Jackson DW, Cozen L. Genu valgum as a complication of proximal tibial metaphyseal fractures in children. J Bone Joint Surg Am. 1971;53:1571-1578.

13. Jenkins DH, Cheng DH, Hodgson AR. Stimulation of bone growth by periosteal stripping. A clinical study. J Bone Joint Surg Br. 1975;57:482-484.

14. Kuijpers-Jagtman AM, Bex JH, Maltha JC, Daggers JG. Longitudinal growth of the rabbit femur after vascular and periosteal interference. Anat Anz. 1988;167:349-358.

15. Limpaphayom N, Prasongchin P. Surgical technique: Lower limb-length equalization by periosteal stripping and periosteal division. Clin Orthop Relat Res. 2011;469:3181-3189.

16. Lynch MC, Taylor JF. Periosteal division and longitudinal growth in the tibia of the rat. J Bone Joint Surg Br. 1987;69:812-816.

17. Noonan KJ, Halanski MA, Leiferman E, Wilsman N. Growth retardation (hemiepiphyseal stapling) and growth acceleration (periosteal resection) as a method to improve guided growth in a lamb model. J Pediatr Orthop. 2015 [Epub ahead of print].

18. Popowics TE, Zhu Z, Herring SW. Mechanical properties of the periosteum in the pig, Sus scrofa. Arch Oral Biol. 2002;47:733-741.

19. Rackard SM, Carr AJ, Callanan JJ, Bellenger CR. An avian model of limb deviation induced by periosteal surgery. Res Vet Sci. 2002;73:237-241.

20. Read EK, Read MR, Townsend HG, Clark CR, Pharr JW, Wilson DG. Effect of hemi-circumferential periosteal transection and elevation in foals with experimentally induced angular limb deformities. J Am Vet Med Assoc. 2002;221:536-540.
21. Sabharwal S, Paley D, Bhave A, Herzenberg JE. Growth patterns after lengthening of congenitally short lower limbs in young children. J Pediatr Orthop. 2000;20:137-145.

22. Sansone JM, Wilsman NJ, Leiferman EM, Noonan KJ. The effect of periosteal resection on tibial growth velocity measured by microtransducer technology in lambs. $J$ Pediatr Orthop. 2009;29:61-67.

23. Shapiro F. Longitudinal growth of the femur and tibia after diaphyseal lengthening. J Bone Joint Surg Am. 1987;69:684-690.

24. Silberman FS, Sola CK, Cabrini RL. A study of the vascular distribution after periosteal stripping of the long bones. Surg Gynecol Obstet. 1967;125:1311-1315.

25. Sola CK, Silberman FS, Cabrini RL. Stimulation of the longitudinal growth of long bones by periosteal stripping. An experimental study on dogs and monkeys. J Bone Joint Surg Am. 1963;45:1679-1684.

26. Stevens PM, Pease F. Hemiepiphysiodesis for posttraumatic tibial valgus. J Pediatr Orthop. 2006;26:385-392.

27. Stokes IA, Aronsson DD, Dimock AN, Cortright V, Beck S. Endochondral growth in growth plates of three species at two anatomical locations modulated by mechanical compression and tension. J Orthop Res. 2006;24:1327-1334.

28. Taylor JF, Warrell E, Evans RA. The response of the rat tibial growth plates to distal periosteal division. J Anat. 1987;151:221-231.

29. Trueta J, Caladias AX. A study of the blood supply of the long bones. Surg Gynecol Obstet. 1964;118:485-498.

30. Uchiyama E, Yamakoshi K, Sasaki T. Measurement of mechanical characteristics of tibial periosteum and evaluation of local differences. J Biomech Eng. 1998;120:85-91.

31. Warrell E, Taylor JF. The role of periosteal tension in the growth of long bones. J Anat. 1979;128:179-184.

32. Wilde GP, Baker GC. Circumferential periosteal release in the treatment of children with leg-length inequality. $J$ Bone Joint Surg Br. 1987;69:817-821.

33. Wilson-MacDonald J, Houghton GR, Bradley J, Morscher E. The relationship between periosteal division and compression or distraction of the growth plate. An experimental study in the rabbit. J Bone Joint Surg Br. 1990;72:303-308.

34. Yabsley RH, Harris WR. The effect of shaft fractures and periosteal stripping on the vascular supply to epiphyseal plates. $J$ Bone Joint Surg Am. 1965;47:551-566. 